\title{
Atuações de um centro educacional e psicológico junto a estudantes universitários ${ }^{1}$
}

\author{
Karolina Murakami ${ }^{2}$ \\ Gisele Curi de Barros \\ Cristiane Martins Peres \\ Rodrigo Humberto Flauzino \\ Maria de Fátima Aveiro Colares \\ Faculdade de Medicina de Ribeirão Preto - Universidade de São Paulo, Ribeirão Preto-SP, Brasil
}

\section{Resumo}

O Centro de Apoio Educacional e Psicológico tem como atribuições o planejamento e execução de programas de apoio e prevenção de dificuldades emocionais e acadêmicas durante a formação do estudante de graduação. Este relato de experiência descreve o funcionamento do CAEP, resgatando sua história e apresentando suas experiências e perspectivas de intervenções na assistência aos estudantes, e assessoria aos docentes e aos cursos. Verifica-se a importância da existência desse centro, que desde sua implementação leva em conta a percepção integral na formação dos futuros profissionais. Neste contexto, as universidades precisam reconhecer suas realidades, de modo a construir estratégias institucionais para ajudar os estudantes a lidarem com os múltiplos desafios que a vivência no ensino superior possa acarretar.

Palavras-chave: Estudante, universidades, educação médica, saúde mental

\section{Abstract: Activities of an educational and psychological center with university students}

The Psychological and Educational Support Center is responsible for the planning and execution of programs to support and prevent emotional and academic difficulties during undergraduate education. This experience report describes the operation of CPES, rescuing the history and presenting experiences and perspectives of interventions in the assistance to students, and advice to teachers and courses. It is emphasized the importance of the existence of this center, which since its implementation considers the integral perception in the training of future professionals. In this context, universities need to recognize their realities in order to build institutional strategies to help students cope with the multiple challenges that higher education experiences may entail.

Keywords: Student, universities, medical education, mental health

\section{Resumen: Acciones de un centro educativo y psicológico para estudiantes universitarios}

El Centro de Apoyo Educacional y Psicológico (CAEP) tiene como atribuciones la planificación y ejecución de programas de apoyo y prevención de dificultades emocionales y académicas durante la formación de estudiantes de grado. Este relato de experiencia describe el funcionamiento del CAEP, retomando su historia y presentando sus experiencias y perspectivas de intervención en la asistencia a los estudiantes y asesoría a los docentes y carreras universitarias. Se constata la importancia de la existencia de este centro que, desde su implementación, tiene en cuenta una percepción integral en la formación de los futuros profesionales. En este contexto, las universidades necesitan reconocer sus realidades para construir estrategias institucionales que auxilien a los estudiantes a lidiar con los múltiples desafíos que se pueden presentar en la enseñanza superior.

Palabras clave: Estudiante; universidades; educación médica, salud mental

\footnotetext{
${ }^{1}$ Agradecimentos: Prof. Dr. Victor Evangelista de Faria Ferraz e Profa. Dra. Maria Paula Panúncio Pinto, coordenador e vice coordenadora do CAEP.

${ }^{2}$ Endereço para correspondência: Centro de Apoio Educacional e Psicológico. Avenida Bandeirantes, 3900, 14049-900, Ribeirão Preto,SP. E-mail: karolina.murakami@usp.br
} 
Os serviços de apoio ao estudante universitário existem em diversas instituições de ensino superior no Brasil e no mundo. No geral, podem contar com profissionais da área de educação (pedagogos e psicopedagogos), da área de saúde mental (psicólogos e psiquiatras), da saúde geral (médicos, dentistas), e assistência social (Serpa \& Santos 2001).

Os estudos que apontam para a importância de tais serviços fazem-no baseados em algumas considerações, tanto em relação ao momento de vida em que o estudante se encontra - final da adolescência, quanto a uma série de questões que envolverão este adulto jovem na relação com o contexto acadêmico e a vida universitária. Sobre a adolescência, surge a preocupação com questões de saúde mental porque alguns transtornos psiquiátricos têm seu surgimento nesta fase do desenvolvimento (Fiorotti, Rossoni, Borges, \& Miranda, 2010; Lima, Domingues, \& Cerqueira, 2006). Há uma produção significativa de trabalhos que abordam tanto a prevalência de transtornos mentais na população universitária, em comparação com a população geral (Adlaf, Gliksman, Demers, \& NewtonTaylor, 2001; Verger, Guagliardo, Gilbert, Rouillon, \& Kovess-Masfety, 2010; Zivin, Eisenberg, Gollust, \& Golberstein, 2009), quanto estudos que trazem a questão do aumento na ingestão de bebida alcoólica e uso de outras substâncias, por conta do ambiente universitário muitas vezes se tornar um facilitador para este tipo de comportamento (Andrade et. al., 2012; Kerr-Corrêa, Andrade, Bassit, \& Boccuto, 1999; Pechansky, Szobot Claud, \& Scivoletto, 2004; Pillon \& Corradi-Webster, 2006; Rocha, Lopes, Martelli, Lima, \& Martelli-Júnior, 2011; Wagner \& Andrade, 2008).

Considera-se também que alguns "sintomas" (crises agudas de ansiedade, sintomas depressivos), embora não configurem propriamente um transtorno psiquiátrico, podem constituir reações de ajustamento nesta etapa que envolve formação de identidade, escolha de carreira, entre outros aspectos. Assim, dado que o ingresso na universidade potencializa a emergência de tais reações, este período da vida dos jovens transformou-se em alvo de estudos, que apontam para o investimento em espaços de acolhimento e manejo destas situações junto aos estudantes, auxiliando-os em uma adaptação que possa ser saudável, evidenciando uma preocupação para além das questões acadêmicas e da formação técnica, priorizando a atenção e os cuidados integrais à população universitária (Bardagi \& Hutz, 2011; Oliveira, Carlotto, Vasconcelos, \& Dias, 2014; Pereira et. al., 2006).

Aspectos da relação do estudante com o contexto acadêmico configuram-se como foco de investigação. Dúvidas quanto à escolha do curso, dificuldades em momentos específicos da formação, relações interpessoais, escolha de especialidades, saída da universidade e ingresso no mercado de trabalho, são temas importantes que merecem destaque (Buscacio \& Soares, 2017).

Podem ser muitos os desafios de uma formação universitária integral, justificando a criação e manutenção de serviços de apoio em vários campi. Especificamente em escolas médicas, o incentivo à criação de espaços de apoio aos seus estudantes também se deu pautada na preocupação com a formação do jovem em uma profissão permeada por exigências técnicas de quem cuidará da vida das pessoas, ficando exposto ao contato constante com a dor, o sofrimento e a morte. Ampliou-se a concepção da importância do cuidado ao próprio estudante em formação, para que este pudesse cuidar integralmente das pessoas submetidas às suas intervenções (Amaral et. al. 2012; Figueiredo \& Oliveira, 1995; Furigo et. al. 2008; Pan, Zonta, \& Tovar, 2015; Oliveira, Dantas, Azevedo, \& Banzato, 2008; Peres, Santos, \& Coelho, 2003; Peres, Santos, \& Coelho, 2004; Pereira et. al., 2006; Pinho, 2016).

Sendo assim, o objetivo deste relato de experiência é descrever os serviços desenvolvidos por um Centro de Apoio Educacional e Psicológico e contextualizar sua existência a partir dos cenários nacional e internacional, e apontar suas perspectivas de atuação integral ao público com o qual interage.

\section{O apoio ao estudante universitário no contexto nacional e internacional}

No âmbito internacional, o cuidado com a saúde mental do estudante universitário começou a ser motivo de preocupação nos países desenvolvidos já no início do século XX. Neste contexto, as instituições de ensino superior passaram a reconhecer que estudantes universitários vivenciam uma fase vulnerável, enfrentando as dificuldades típicas do desenvolvimento que acompanham o final da adolescência e início da idade adulta, além dos desafios do ensino superior. Entendia-se que a responsabilidade em ajudá-los naquele momento seria propriamente da instituição que estavam inseridos. Assim, na década de 1950, muitos serviços de saúde mental foram fundados em universidades nos Estados Unidos e na Europa (Cerchiari, Caetano, \& Faccenda 2005; Oliveira et. al., 2008).

No contexto brasileiro, a saúde mental dos estudantes de medicina tem sido foco de pesquisa desde a década de 60. Segundo Baldassin (2012), os primeiros serviços de assistência destinados à saúde mental dos estudantes de medicina foram o Centro de Atendimento da Psicologia (Ceap), criado em 1966 na Universidade Federal de Minas Gerais; o Serviço de Benefício ao Estudante (Sebe), 
implementado na Universidade Federal do Piauí em 1976, e a Coordenação de Assistência e Integração do Estudante (Codae), estruturado em 1980 na Universidade Federal de Sergipe. Ainda de acordo com este autor, o primeiro artigo sobre o assunto ("Sobre problemas de higiene mental dos universitários") foi atribuído ao psiquiatra Galdino Loreto, da Faculdade de Medicina da Universidade de Pernambuco.

Em seu trabalho, Baldassin (2012) catalogou 164 escolas de medicina no Brasil. Dessas, 128 (78\%) responderam a solicitação desse autor para colaborar em sua pesquisa com dados informativos sobre serviços de assistência aos estudantes nas escolas médicas. Evidenciou-se que $65(51 \%)$ afirmaram ter um serviço de atendimento psicológico ou pedagógico aos estudantes. Destas 65 escolas, $66 \%$ eram particulares e $34 \%$ públicas. A maior proporção de escolas que possuíam o serviço estava nas regiões Sul e Sudeste, e a menor proporção estava nas regiões Norte e Centro-Oeste. Os Estados brasileiros com maior número de serviços foram São Paulo (18 - 28\% das escolas), Minas Gerais e Rio Grande do Sul (nove cada $14 \%$ das escolas). Os grupos que atendiam os estudantes eram constituídos, em sua maioria, por psicólogos, psicopedagogos e assistentes sociais. A maioria dos serviços não atendia exclusivamente estudantes de Medicina, e foram criados após o ano 2000.

O Fórum de Pró-Reitores de Assuntos Comunitários e Estudantis (FONAPRACE) realizou, em 1999 e 2000, um mapeamento sobre a assistência praticada nas instituições públicas brasileiras de ensino superior, entre 40 instituições federais e estaduais. Levantaram-se vários itens, dentre eles, os programas de saúde oferecidos: atendimentos médicos, exames para diagnóstico, atendimento odontológico, atendimento psicológico/ psiquiátrico, prevenção de dependência química e tratamento de dependência química. Verificou-se que das 40 instituições, 34 (85\%) ofereciam algum tipo de atendimento à saúde mental do estudante universitário (Cerchiali et al. 2005).

A implantação dos serviços de apoio aos estudantes universitários é ponderada pela vulnerabilidade dessa população. As exigências da etapa de formação profissional, especialmente nos cursos da área de saúde, fazem com que eles precisem se ajustar e se adaptar a muitas transformações. Há estudantes que podem atravessar tais mudanças sem dificuldades no aprendizado e desenvolvimento. Outros podem vivenciar um grande sofrimento psíquico, podendo resultar em impacto nos relacionamentos e na qualidade de vida, comprometer o desempenho em atividades diárias, incluindo as acadêmicas, e ser potencial substrato para o desenvolvimento de transtornos mais graves (Colares \& Andrade, 2009; Panúncio-Pinto \& Colares, 2015). Além disso, sabe-se que o desenvolvimento e a manutenção de determinados componentes adaptativos dos estudantes durante o curso da área da saúde podem influenciar a qualidade do cuidado dado aos pacientes no exercício da profissão (Miranda, Pires, Nassar, \& Silva, 2009; Troncon et al., 2013).

Tempsky e Martins (2012) afirmam que as escolas médicas, além de terem a responsabilidade de formar médicos, devem garantir que esta formação não danifique a saúde física, mental e social desta população. As autoras apontam para a melhoria da qualidade de vida dos estudantes através medidas como: mudanças curriculares (que garantam tempo livre para estudos e outras atividades); esforço para diminuir a competitividade; combate à auto cobrança por meio de grupos de discussão, tutoria e grupos de suporte; apoio psicológico, pedagógico e Tutoria Mentoring; manutenção de programas de promoção da saúde com estímulo à alimentação saudável e exercício físico, trabalho evolvendo gestão de tempo (Tempsky \& Martins, 2012).

Considera-se que melhorar a percepção de qualidade de vida no ensino superior depende de medidas de desenvolvimento pessoal e de desenvolvimento institucional. Auxiliar os estudantes a cuidar de sua saúde física e mental, a estabelecer e manter seus relacionamentos, gerir seu tempo e desenvolver resiliência, torna-se importante para que eles possam amadurecer minimizando possíveis dificuldades durante o percurso universitário. Neste sentido, o CAEP tem desenvolvido um importante trabalho nestes aspectos.

\section{O Centro de Apoio Educacional e Psicológico: histórico e criação}

O Centro de Apoio Educacional e Psicológico (CAEP) foi criado dentro de uma instituição pública universitária, em 1990, a partir de demandas apresentadas pela Comissão de Graduação (CG) com questões relacionadas à saúde mental do estudante de medicina. As discussões iniciais para implantação do serviço foram intensificadas ao se verificar a necessidade de atenção às dificuldades emocionais e acadêmicas vivenciadas pelos alunos ao longo do curso.

Embora a origem dos questionamentos e reflexões da instituição acerca da criação de um serviço de suporte aos estudantes estivesse relacionada, em primeira instância, às demandas de natureza psicológica e psiquiátrica ocorridas nesta população, havia também uma grande preocupação em dar suporte pedagógico à gestão do ensino de graduação. Portanto, o serviço deveria ser instituído de forma a 
considerar a formação acadêmica do estudante como algo integral, abrangendo os aspectos psicológicos e também os psicoeducacionais, voltados para o auxílio a uma melhor aprendizagem no ambiente universitário (Cianflone, Figueiredo \& Colares, 2002). Assim, foi definido o princípio básico deste Centro: a atuação deveria se voltar tanto ao estudante e suas eventuais dificuldades, como a todo o campo institucional.

$\mathrm{Na}$ época da criação do CAEP, a Faculdade pública na qual ele se vincula, contava apenas com o curso de Medicina. Dessa forma, todas as diretrizes que nortearam a inauguração do mesmo pautaram-se em aspectos relacionados à formação médica no geral. A partir de 2002, o serviço passou a atender as demandas dos diferentes cursos que foram sendo implantados, a saber, Fisioterapia, Fonoaudiologia, Nutrição, Terapia Ocupacional, Informática Biomédica e Ciências Biomédicas.

As atividades realizadas pelo CAEP se baseiam em três pilares: ensino, como prestação de assessoria à Comissão de Graduação (CG) e aos docentes da Faculdade pesquisa, com o desenvolvimento de projetos e pesquisa sobre educação nas profissões da saúde, caracterização pedagógica, psicossocial e psicopedagógica de sua população estudantil; e assistência, como desenvolvimento de atividades de suporte psicopedagógico e psicológico aos alunos do curso de graduação, com ênfase na identificação das características acadêmicas, emocionais e na prevenção de dificuldades de aprendizagem e/ou psicológicas.

A estrutura do CAEP permite a atuação de dois setores distintos (Psicologia e Educação) que se inter-relacionam em suas funções. Sua equipe técnica é composta atualmente por três psicólogas, uma psicopedagoga, um pedagogo e uma técnica administrativa. A gestão e administração do serviço são conduzidas por um grupo de consultores, constituído por docentes indicados pela Comissão de Graduação (CG), à qual o Centro é subordinado. Eles são responsáveis pela interlocução entre a equipe técnica e os demais membros da instituição, com o intuito de colaborar na decisão sobre as principais diretrizes do serviço e, também, para refletir sobre situações complexas que exigissem análises específicas. Além do grupo de consultores, há também um coordenador, que tem como funções traçar diretrizes, supervisionar as atividades desenvolvidas e deliberar sobre solicitações encaminhadas ao CAEP.

Através de seu processo histórico, observa-se que toda a construção do CAEP, assim como dos demais serviços de apoio, teve como foco principal auxiliar o estudante no seu desenvolvimento pessoal e profissional, no decorrer de sua formação acadêmica.

\section{O caráter do serviço e suas demandas institucionais:}

\section{Assistência Psicológica e Educacional aos estudantes}

O Centro de Apoio Educacional e Psicológico (CAEP), atuante desde 1990, elabora periodicamente relatórios contendo suas atividades e demandas efetuadas. Para este relato de experiência, foram analisados os registros entre os anos de 2014 a 2017 sobre as características demográficas dos estudantes e os atendimentos individuais realizados. A sistematização e a utilização dos dados assistenciais, colhidas por meio de programas e ações do CAEP, foram submetidas ao Comitê de Ética em Pesquisa, sendo aprovadas em sua $425^{\mathrm{a}}$ reunião ordinária, como consta no processo HCRP $\mathrm{n}^{\circ} 1785 / 2016$.

Ao longo do período considerado, 655 estudantes buscaram espontaneamente o serviço com demandas individuais. Após o primeiro contato, eles foram encaminhados para seguintes modalidades: (a) triagem psicológica, (b) psicoterapia breve, (c) orientação educacional (psicopedagógica e/ou pedagógica), o que gerou um total de 3.996 atendimentos. Tais modalidades de atendimento são apresentadas e descritas abaixo:

1. A triagem psicológica consiste em uma entrevista com o intuito de conhecer a demanda que motivou o estudante a buscar assistência psicológica, avaliar sinais, sintomas, gravidade da situação (existência de risco), e determinar uma conduta (psicoterapia, encaminhamento para outros serviços, etc.).

2. A psicoterapia breve é o seguimento feito com o profissional psicólogo, oferecido aos estudantes através de procura espontânea. Após algumas entrevistas de avaliação (triagem), se houver indicação, realiza-se o atendimento psicoterápico, geralmente com sessões semanais individuais.

3. O acompanhamento educacional (psicopedagógico e/ou pedagógico) - o conceito que embasa tal modalidade é o da metacognição, que trata da tomada de consciência das estratégias que cada um usa para aprender. A eficácia da aprendizagem depende da construção de estratégias cognitivas que possibilitem ao indivíduo planejar e monitorar o seu desempenho. Esse processo pode ainda exercer influência sobre a motivação, pois o fato dos estudantes poderem controlar e gerir o seu próprio aprendizado lhes dá a noção da responsabilidade pelo seu desempenho escolar e gera confiança nas suas próprias capacidades.

A seguir, será apresentada a Tabela 1, que descreve o número de atendimentos realizados em cada modalidade descrita acima entre os anos 2014 a 2017. 
Tabela 1

Número de atendimentos realizados em cada modalidade, 2014-2017

\begin{tabular}{ccccc}
\hline Anos & $\begin{array}{c}\text { Triagem } \\
\text { Psicológica }\end{array}$ & $\begin{array}{c}\text { Psicoterapia } \\
\text { Breve }\end{array}$ & $\begin{array}{c}\text { Orientação } \\
\text { Psicopedagógica }\end{array}$ & $\begin{array}{c}\text { Total de atendimentos } \\
\text { realizados }\end{array}$ \\
\hline 2014 & 104 & 832 & 82 & 1018 \\
2015 & 80 & 1169 & 115 & 1364 \\
2016 & 108 & 793 & 125 & 1026 \\
2017 & 73 & 454 & $61^{1}$ & 588 \\
Total & 365 & 3248 & 383 & 3996 \\
\hline
\end{tabular}

${ }^{1}$ Além dos 61 atendimentos psicopedagógicos, no segundo semestre de 2017, houve agregação de mais um membro pedagogo à equipe do CAEP, que ficou responsável por alguns acompanhamentos individuais, com caráter de orientação pedagógica. Ao todo, foram realizadas 9 sessões, especificamente voltadas à organização de estudos e rotinas

Dos alunos, 365 estudantes foram atendidos especificamente na triagem psicológica (coluna 2). Algumas características da demanda se destacam e serão apresentadas a seguir.

Os alunos atendidos pelo serviço são jovens, no início da vida adulta, especialmente na faixa entre 20 a 22 anos (45\%). Há predomínio de mulheres $(73 \%)$ em relação aos homens, e segundo as diferentes etapas da graduação, a procura pelo serviço é maior na etapa inicial do curso (43\% correspondem ao $1^{\circ}$ e ao $2^{\circ}$ anos no curso de Medicina, Fisioterapia, Nutrição e Terapia Ocupacional, e $1^{\circ}$ ano dos cursos de Informática Biomédica, Fonoaudiologia e Ciências Biomédicas).

As queixas mais frequentes apresentadas pelos estudantes que procuraram o CAEP de 2014 a 2017 para atendimento psicológico foram: sintomas de transtornos de ansiedade; sintomas de depressão; sintomas de transtorno de ajustamento; dificuldades de relacionamento interpessoal; dificuldades acadêmicas e dúvidas vocacionais.

Como revela a Tabela 1, foram realizadas mais sessões de Psicoterapia Breve em comparação aos outros tipos de atendimento do serviço, no referido período (total de 3248 sessões). Esse fato ocorre, principalmente, porque o número de profissionais da área da psicologia é maior, bem como o processo terapêutico, ainda que seja a abordagem da Terapia Breve, dura em torno de seis meses a 1 ano, com frequência semanal.

No que tange ao atendimento psicopedagógico individual, as queixas que levaram os estudantes a buscar o CAEP no referido período relacionaram-se a: baixo rendimento escolar; organização da rotina de estudos; dificuldade de concentração; ansiedade escolar; insatisfação com o curso; escolha profissional; dificuldades de aprendizagem; dificuldades interpessoais.

Considerando o total de vagas oferecidas anualmente para os estudantes dos sete cursos da Faculdade pública em questão, tem-se 1530 alunos matriculados. Analisando a demanda por atendimentos nas referidas modalidades (triagem, psicoterapia breve e orientação psicopedagógica) oferecidas pelo CAEP nos últimos quatro anos, registrou-se que, em média, $11 \%$ da população de estudantes procuraram o serviço e foram atendidos por seus profissionais. A tabela 2 apresenta o número de graduandos que espontaneamente buscou apoio às suas demandas individuais, no período de 2014 a 2017.

Tabela 2

Graduandos que buscaram apoio e seu percentual em relação às matrículas

\begin{tabular}{lll}
\hline Anos & $\mathrm{N}^{\circ}$ & $\%$ \\
\hline 2014 & 175 & 11 \\
2015 & 173 & 11 \\
2016 & 202 & 13 \\
2017 & 105 & 7 \\
Total & 655 & 11 \\
\hline
\end{tabular}

Em 2014, foram atendidos 175 alunos (11\% dos estudantes da Faculdade em questão). A porcentagem de estudantes atendidos pelo serviço permaneceu a mesma no ano de 2015 (11\%). Em 2016 a demanda aumentou, 202 estudantes solicitaram atendimento no CAEP (13\%), e em 2017 foram atendidos 105 alunos (7\%). Este último marcou o início de um ano de transição, em que mudanças na estruturação do serviço começaram a ocorrer.

Com o intuito de expandir a atuação do atendimento do CAEP, para além do âmbito individual (que, embora significativo, ao longo da história do serviço mostrou-se limitado a uma pequena parcela da população estudantil da Faculdade), o Centro procurou enfatizar a estruturação de ações grupais, mantendo intervenções já consolidadas nesse aspecto, e dando um novo delineamento às perspectivas de intervenção voltadas à promoção e à prevenção da saúde mental e, consequentemente, à melhoria da qualidade de vida na universidade. 
No segundo semestre de 2017, foi implantado no serviço o aconselhamento psicológico, uma nova modalidade de atendimento que visa substituir gradualmente o modelo de psicoterapia breve. O aconselhamento psicológico é um atendimento dirigido a situações contextuais, que permite aos estudantes trabalharem as dificuldades que vêm enfrentando através de seus próprios recursos, em um curto espaço de tempo. Pode ser realizado em até 04 sessões. Nesta modalidade de atendimento o trabalho é direcionado, focado para a resolução de problemas, tomada de decisões e autoconhecimento (Scorsolini-Comin, 2014). Durante o desenvolvimento destes encontros, havendo demandas específicas, podem ocorrer encaminhamentos para outros serviços do CAEP ou serviços de apoio público.

\section{Programas de Apoio ao estudante}

Além dos atendimentos individuais realizados pelos profissionais dos setores de Psicologia e Educação, a promoção de atividades coletivas tem sido um pilar importante para dar apoio ao estudante em sua trajetória acadêmica. Dentre essas atividades, destacam-se:

\section{Programa de Tutoria}

O programa de Tutoria na categoria Mentoring é uma atividade de suporte e recepção aos ingressantes (calouros) dos cursos da Faculdade, desenvolvida por tutores (mentores) e adotada em escolas médicas no Brasil e no mundo (Bellodi \& Martins, 2005; Bellodi, 2007). Nesta Faculdade pública, o referido programa foi instituído por meio do CAEP em 2003, inicialmente junto ao curso da Medicina e posteriormente os sete cursos da instituição foram aderindo ao Programa. Desde o princípio, a iniciativa de implantação do Programa foi organizada e executada pelo CAEP, com apoio da Comissão de Graduação e Diretoria da Faculdade com o propósito de favorecer o acolhimento e a adaptação do estudante ao novo contexto universitário. Os tutores são docentes da Faculdade, profissionais contratados do Hospital das Clínicas e pós-graduandos da Faculdade, que tenham envolvimento acadêmico com os estudantes e cuja formação profissional seja afim com o respectivo curso no qual atuam. Através de alguns encontros grupais, os tutores recebem uma capacitação inicial, oferecido pela equipe técnica do CAEP. O intuito da capacitação é proporcionar aos Tutores um melhor entendimento dos objetivos do Programa e de sua função enquanto "mentores". Os encontros de tutoria são quinzenais, e ocorrem ao longo do primeiro semestre de cada ano.
Além da participação de tutores, o Programa também contempla a participação de alunos veteranos que atuam como parceiros. A "Tutoria por pares", comumente encontrada na literatura inglesa como Peer Tutoring, tem sido uma estratégia bastante utilizada em cursos de nível superior e permite que alunos veteranos possam contribuir com os calouros, compartilhando suas experiências relativas à formação acadêmica (Cardozo-Ortiz, 2011; Simão, Flores, Fernandes, \& Figueira, 2008). Os pares possuem uma importância significativa nessa atividade, pois podem auxiliar o tutor em diversas atribuições durante os encontros do Programa.

O Programa de Tutoria Mentoring da faculdade tem como principais objetivos: estimular a integração e adaptação dos calouros ao início da vida acadêmica; identificar as motivações do estudante para a profissão e dirigi-las adequadamente; ajudar o estudante a ter uma perspectiva real sobre a prática profissional; facilitar o estabelecimento de relações interpessoais no período inicial do curso (com veteranos e professores); oferecer informações acadêmicas gerais sobre início da vida acadêmica.

Ao longo dos anos, o desenvolvimento dessa abordagem tem se revelado muito profícua, uma vez que as ações tutoriais (AT) são compatíveis com o projeto educacional da instituição, tanto do ponto de vista da orientação pessoal, quanto da orientação acadêmica ao estudante. A tutoria contribui para proporcionar ao aluno uma formação integral, auxilia em sua adaptação e nas iniciais tomadas de decisão. Além disso, colabora para que ele lide ou até supere dificuldades na forma de estudar e possa receber uma orientação mais direta quanto às aptidões, habilidades e interesses relacionados ao mundo profissional.

\section{As Oficinas de orientações psicopedagógica/pedagógica}

A organização de atividades em grupos, nas quais os alunos têm a oportunidade de ampliar seus olhares e percepções sobre o dia a dia da vida universitária, tem-se constituído uma grande estratégia para ajudá-los a lidar com potenciais situações estressoras. Uma das principais atividades de prevenção, realizada pela psicopedagoga, é aquela que busca identificar os estilos de aprendizagem (Felder \& Silverman, 1998) dos estudantes ingressantes, e a partir disso, discutir estratégias de estudo que mais se adéquem ao estilo de cada um e à singularidade de cada disciplina.

Essas situações ocorrem preferencialmente no $1^{\circ}$ semestre de cada ano escolar, em formato de Oficina intitulada Meu estilo de aprendizagem, cuja duração é de uma hora e meia. Com a chegada de um novo pedagogo à equipe do CAEP em 2017, as oficinas passaram a ser 
ampliadas, o que possibilitou a oferta de outros encontros junto ao público universitário com a finalidade de contribuir para o autoconhecimento das potencialidades e dificuldades de cada um, ampliação dos repertórios sobre formas de estudar, organização da rotina, desenvolvimento da memória, dúvidas sobre a escolha profissional, enfim, temas que emergem continuamente em atendimentos individuais. A maioria das oficinas que tem surgido, seguem um modelo interdisciplinar e interprofissional, já que coloca estudantes de vários cursos e anos juntos. Seguindo modelos educacionais mais atuais, centrados no estudante, o papel mais ativo dos estudantes nos processos de mudança que advém basicamente da troca de experiências entre os parceiros, tem substituído aos poucos a postura mais assistencial do serviço (Peres, Vieira, Altafim, Mello, $\&$ Suen, 2014; Ausubel, 2000).

Parcerias com professores que possuem expertise em determinados temas têm ocorrido com frequência no serviço. Alguns exemplos: uma docente da Fonoaudiologia, que trabalha com a temática do falar em público, desenvolveu uma oficina; um docente da Neurofisiologia, que estuda memória, teve importante participação em um mini curso sobre organização dos estudos. Assim, têm-se fortalecido as relações entre professores e alunos, bem como a confiança no potencial de comunidades colaborativas no contexto acadêmico.

\section{Grupos de reflexão}

São grupos que podem ser formados a partir de temas propostos pelos próprios estudantes, geralmente relacionados ao desenvolvimento da identidade profissional. Os grupos reflexivos apresentam-se como uma estratégia profilática que permite aos estudantes vivenciarem e compartilharem em grupo suas angústias, medos e incertezas, em torno de situações que são comuns a todos, podendo levar a reflexões mais positivas e críticas frente ao papel profissional que está em desenvolvimento. No curso de Nutrição e Metabolismo, por exemplo, o setor de Psicologia do CAEP vem desenvolvendo encontros nesta modalidade de intervenção junto aos alunos que estão passando pelos estágios do último ano da formação.

\section{Divulgação do serviço na matrícula anual dos calouros}

O CAEP tem participação ativa na matrícula dos calouros dos cursos geridos pela Faculdade, com o objetivo de orientar estudantes e familiares sobre as várias atividades de suporte psicológico, psicopedagógico e pedagógico desenvolvido pelo serviço. Construir esse laço de confiança e acolhimento já nesse primeiro contato do ingressante com a universidade tem fortalecido ainda mais a rede de apoio, de cuidado e de atenção à qualidade de vida.

\section{Apoio aos docentes e comissões}

Assessoria pedagógica e psicopedagógica à Comissão de Graduação e às Comissões Coordenadoras de Curso

No âmbito das atribuições do setor educacional, encontra-se a participação mensal de seus educadores (psicopedagoga e pedagogo) como membros convidados nas Comissões Organizadoras dos Cursos de Graduação (COCs) bem como junto à Comissão de Graduação $(\mathrm{CG})$ da faculdade. O papel desses profissionais é o de fornecer subsídios, assessorar, contribuir com as discussões e decisões acerca do universo pedagógico que circunda os temas abordados em cada colegiado (avaliação do estudante, das disciplinas, do curso, organização de estratégias de ensino, entre outras demandas). Regularmente, as COCs também solicitam a participação dos educadores em grupos de trabalho ou em reuniões específicas, para ajudar a tratar de algum tema ou orientar quanto a ações que podem ser realizadas com determinado estudante ou docente, a partir do contexto analisado.

Ainda sobre a participação do CAEP como apoio educacional, destaca-se a inserção de seus membros em disciplinas da graduação, colaborando com a formação acadêmica e profissional dos estudantes, a partir de uma perspectiva ampla de atuação sobre matriz curricular, metodologias de aprendizagem ou outras questões ligadas à missão de cada curso. Também se destaca a contribuição técnica e pedagógica em Fóruns de Departamentos e/ou cursos, e em discussões e encaminhamentos acerca de eventuais ajustes em disciplinas e currículos.

\section{Grupos focais}

Com uma perspectiva voltada a dinâmicas com grupos para diversos fins pedagógicos, o setor educacional tem promovido avaliações e análise de diversas temáticas por meio da técnica com grupos focais. O referencial de apoio para as construções dos roteiros tem sido o modelo proposto por Peres (2006), que deriva das contribuições do Sociodrama Educacional (Romaña, 1996). Os profissionais desse setor organizam grupos de docentes ou estudantes que podem ser formados a partir de temas propostos pelas demandas institucionais (geralmente relacionadas a mudanças curriculares e/ou avaliação de disciplinas). $\mathrm{Na}$ abordagem de entrevista grupo focal, como sugerem as 
abordagens de investigação qualitativa, os entrevistadores assumem o papel de facilitadores e moderadores das discussões, e os dados são trazidos pela interação grupal que se desenvolve ao redor daquilo que foi previamente construído junto com as pessoas que solicitaram o uso dessa estratégia (Morgan, 1997). Quanto à operacionalização do grupo focal, o registro de áudio dos relatos dos participantes e sua transcrição são fundamentais para permitir que as técnicas de análise sejam aplicadas de forma fidedigna e para que possa ser realizada por pesquisadores independentes, caso seja prerrogativa do desenho metodológico do estudo.

\section{Participação nas Comissões Institucionais}

Comissão de Avaliação - Regularmente em reuniões mensais, os educadores do CAEP também têm prestado assessoria, no que concerne ao âmbito de questões pedagógicas, ao Centro de Avaliação do Ensino de Graduação, cuja comissão institucional é formada por um grupo de docentes e discentes. A missão desse outro centro é oferecer subsídios e dar suporte à Comissão de Graduação (CG), e às Comissões Coordenadoras de Curso da Faculdade, no que se refere ao desenho, implementação e monitoramento dos sistemas de avaliação das disciplinas, principalmente do estudante nos cursos de graduação da faculdade.

Comissão de Direitos Humanos - Criada no dia 05 de agosto de 2016, através da Portaria 45/2016, a Comissão de Direitos Humanos da faculdade de Medicina tem por objetivo nortear as relações humanas em um ambiente plural e de respeito, promovendo o humanismo no ambiente acadêmico com especial atenção no combate à violência interpessoal, à discriminação de gênero, raça, cor, etnia, religião, idade, classe social, orientação sexual e identidade de gênero, entre outras. A Comissão conta com a participação de docentes, discentes e funcionárias. Dos membros do CAEP participam da comissão uma psicóloga, a técnica administrativa, o coordenador e a vice-coordenadora do Centro.

\section{A pesquisa como fundamentação das práticas do serviço}

O serviço tem investido na organização de um banco de dados sobre caracterização pedagógica, psicossocial e psicopedagógica de sua população estudantil. Compreender como aspectos sociais e psicológicos afetam o desenvolvimento acadêmico e a capacidade do estudante para a aquisição de habilidades e competências ao longo da graduação é uma meta importante, embora difícil de alcançar, e que ganha relevância para a equipe do serviço e para a gestão da faculdade, uma vez que dados sobre a evolução dos estudantes são fundamentais para subsidiar o planejamento educacional, em qualquer escola. Sendo assim, cada vez mais tem se investido na formação continua dos profissionais do serviço para que o caráter de um centro de estudos ganhe cada vez mais relevância no contexto acadêmico da referida faculdade.

\section{Considerações finais}

Este relato de experiência buscou descrever o funcionamento do Centro de Apoio Educacional e Psicológico (CAEP), resgatando sua história dentro dos cenários nacional e internacional da criação de serviços de apoio aos estudantes do ensino superior, sobretudo aqueles ligados aos cursos da área de saúde. Descreveu ainda suas experiências na assistência psicológica e educacional ao estudante, bem como as diversas assessorias aos docentes e cursos. Apresentou um recorte de dados dos atendimentos realizados entre 2014 a 2017, revelando o quanto tem sido importante a existência de um centro de apoio que, desde sua implementação, possui características que levam em conta a percepção integral na formação dos futuros profissionais. Por fim, apresentou seu leque de modalidades de atendimentos individuais e coletivos, e apoio ao ensino e à pesquisa, dentro de suas possibilidades e limites de atuação.

Toda essa articulação tem feito com que o CAEP se consolide de forma gradual, reflexiva e permanente como um serviço com enfoque global ao contexto universitário e com respeito às vozes e olhares dos muitos atores envolvidos nesses processos. Entretanto, segundo Behrens (2009), os atores que fazem parte deste cenário compreendem que "a visão de terminalidade oferecida na graduação precisa ser ultrapassada" (p. 70), já que o grau de complexidade e de volume do conhecimento produzido atualmente nunca chegará a ser completamente acessado tanto pelo estudante quanto pelo docente. Se a instituição não se debruçar diante deste fato, e reconhecer que o professor atualmente tem o papel de mediador, e não mais de detentor do saber, pode-se ter continuamente um gerador de ansiedade. Nessa direção, o serviço tem se empenhado na promoção de discussões sobre o planejamento do ensino de graduação, sobre as melhores estratégias para o aprendizado do estudante em todos os domínios do conhecimento profissional, seja ele cognitivo, procedimental ou atitudinal e para que os programas previstos melhor respeitem às necessidades integrais dos alunos (Demo, 2011).

Divulgar o êxito dessas experiências faz parte do compromisso social do CAEP com a sociedade. As 
universidades precisam reconhecer suas realidades, de modo a construir estratégias institucionais que orientem os estudantes a lidar com os múltiplos desafios que a vivência no ensino superior possa acarretar.

\section{Referências}

Adlaf, E. M., Gliksman, L., Demers, A., \& Newton-Taylor, B. (2001). The prevalence of elevated psychological distress among Canadian undergraduates: findings from the 1998 Canadian Campus Survey. Journal of American College Health, 50(2), 67-72.

Amaral, A. E. V., Silva M. A., Lopes, F. L., Leite, C. A., Luca, L., \& Rodrigues, T.C. (2012). Programa de acolhimento a universitários: relato de experiência em clínica-escola. Revista de Psicologia, 15(23), 27-35.

Andrade, A. G., Duarte, P. do C. A. V., Barroso L. P., Nishimura R., Alberghini D. G., \& Oliveira, L. G. (2012). Use of alcohol and other drugs among Brazilian college students: effects of gender and age. Revista Brasileira de Psiquiatria, 34(3), 294-305.

Ausubel D. P. (2000). The acquisition and retention of knowledge: a cognitive view. Dordrecht, Netherlands: Kluwer Academic Publishers.

Baldassin, S. (2012). Quem atende os estudantes de Medicina no Brasil? In: S. Baldassin (Coord.), Atendimento psicológico aos estudantes de medicina - Técnica e ética (pp. 17-22). São Paulo: Edipro.

Bardagi, M., \& Hutz, C. (2011). Eventos estressores no contexto acadêmico: uma breve revisão de literatura brasileira. Inteiração em Psicologia, 15(1), 111-119.

Behrens, M. A. (2009). Projetos de aprendizagem colaborativa num paradigma emergente. In: E. Moran, J. M. Masseto, \& M. A. Behrens. Novas tecnologias e mediação pedagógica, Campinas: Papirus.

Bellodi, P. L (2007). Retaguarda emocional para o aluno de medicina da Santa Casa de São Paulo. Revista Brasileira de Educação Médica, 31(1), 5-14.

Bellodi, P. L., \& Martins, M. A. (2005). Tutoria Mentoring na formação médica. São Paulo: Casa do Psicólogo.

Buscacio, R. C. Z., \& Soares, A. B. (2017). Expectativas sobre o desenvolvimento da carreira em estudantes universitários. Revista Brasileira de Orientação Profissional, 18(1), 69-79.

Cardozo-Ortiz, C. E., (2011). Tutoria entre pares como una estratégia pedagógica universitaria. Educ. Educ., 14(2), 309-325.

Cerchiari, E. A. N., Caetano, D., \& Faccenda, O. (2005). Utilização do serviço de saúde mental em uma universidade pública. Psicologia: Ciência e Profissão, 25(2), 252-265.

Cianflone, A.R. L., Figueiredo, J.F.C., \& Colares, M.F.A. (2002). O Centro de Apoio Educacional e Psicológico (CAEP) da Faculdade de Medicina de Ribeirão Preto (USP): História e perspectivas. Medicina (Ribeirão Preto. Online), 35(3), 392-396.

Colares, M. F. A., \& Andrade, A.S. (2009). Atividades grupais reflexivas com estudantes de medicina. Revista Brasileira de Educação Médica, 33(1), 101-114.

Demo, P. (2011). Olhar do educador e novas tecnologias. Boletim Técnico do SENAC, 37(2), 15-26.

Felder, R. M., \& Silverman, L. K. (1998). Learning and teaching styles in engineering education. Engineering Education, 78(7), 674-681.

Figueiredo R. M., \& Oliveira M. A. P. (1995). Necessidades de estudantes universitários para implantação de um serviço de orientação e educação em saúde mental. Revista Latino-Americana de Enfermagem, 3(1), 05-14.

Fiorotti, K. P., Rossoni R. R., Borges L. H., \& Miranda A. E. (2018). Transtornos mentais comuns entre os estudantes do curso de medicina: prevalência e fatores associados. Jornal Brasileiro de Psiquiatria, 59(1), 17-23.

FMRP-USP. Faculdade de Medicina de Ribeirão Preto da Universidade de São Paulo. Centro de Apoio Educacional e Psicológico. (2014a). Recuperado em 29 de janeiro de 2018, de http://www.fmrp.usp.br/a-faculdade/ estrutura-administrativa/centros-de-apoio/site-caep.

FMRP-USP. Faculdade de Medicina de Ribeirão Preto da Universidade de São Paulo. Centro de Avaliação no Ensino de Graduação. (2014b). Recuperado em 08 de fevereiro de 2018, de http://www.fmrp.usp.br/wp-content/uploads/ Regimento-Interno-CAEG.pdf.

Furigo, R. C. P. L., Sampedro, K. M., Zanelato, L. S., Foloni, R. F., Ballalai, R. C., \& Ormrod, T.. (2008). Plantão psicológico: uma prática que se consolida. Boletim de Psicologia, 58(129), 185-192.

Kerr-Corrêa, F., Andrade, A, G., Bassit, A. Z., \& Boccuto, N. M. V. F. (1999). Uso de álcool e drogas por estudantes de medicina da Unesp. Revista Brasileira de Psiquiatria, 21(2), 95-100. 
Lima, M. C. P., Domingues, M. S., \& Cerqueira, A. T. A. R. (2006). Prevalência e fatores de risco para transtornos mentais comuns entre estudantes de medicina. Revista de Saúde Pública, 40(6), 1035-1041.

Miranda, S. M., Pires, M. M. S., Nassar, S. M., \& Silva, C. A. J. (2009). Construção de uma escala para avaliar atitudes de estudantes de medicina. Revista brasileira de educação médica, 33(1), 104-110.

Morgan D. L. (1997). Focus groups as qualitative research (2nd. Ed). California: Sage Publications.

Oliveira, C. T., C., Carlotto, R. C., Vasconcelos, S. J. L., \& Dias, A. C. G. (2014). Adaptação acadêmica e coping em estudantes universitários brasileiros: uma revisão de literatura. Revista Brasileira de Orientação Profissional, 15(2), 177-186.

Oliveira M. L. C., Dantas, C. R., Azevedo, R. C. S., \& Banzato C. E. M. (2008). Demographics and complaints of university students who sought help at a campus mental health service between 1987 and 2004. São Paulo Medical Journal, 126(1), 58-62.

Pan, M., Zonta, G.A., \& Tovar, A. (2015). Plantão institucional: relato de experiência de uma intervenção psicológica na UFPR. Psicologia em estudo, 20(4), 555-562.

Panúncio-Pinto, M. P. \& Colares, M. F. A. (2015). O estudante universitário: os desafios de uma educação integral. Medicina (Ribeirão Preto), 48(3), 273-81.

Pechansky, F., Szobot, C. M., \& Scivoletto, S. (2004). Uso de álcool entre adolescentes: conceitos, características epidemiológicas e fatores etiopatogênicos. Revista Brasileira de Psiquiatria, 26(1), 14-17.

Pereira, A. M. S., Motta, E. D., Vaz, A. L., Pinto, C., Bernardino, O., Melo, A. C., Ferreira, J., Rodrigues, M. J., Medeiros, A., \& Lopes, P. N. (2006). Sucesso e desenvolvimento psicológico no Ensino Superior: Estratégias de intervenção. Análise Psicológica, 24(1), 51-59.

Peres, C. M. (2006). Atividades extracurriculares: percepções e vivências durante a formação médica. Dissertação de Mestrado. Faculdade de Filosofia Ciências e Letras da Universidade de São Paulo, Ribeirão Preto, São Paulo.

Peres, C. M.; Vieira, M. N. C. M; Altafim, E. R. P.; Mello, M. B., \& Suen, K.S. (2014). Abordagens pedagógicas e sua relação com as teorias de aprendizagem. Revista Medicina (Ribeirão Preto), 47(3), 249-55

Peres, R. S., Santos, M. A, \& Coelho, H. M. B. (2003). Atendimento psicológico a estudantes universitários: considerações acerca de uma experiência em clínica-escola. Estudos de Psicologia (Campinas), 20(3), 47-57.

Peres, R. S., Santos, M. A., \& Coelho, H. M. B. (2004). Perfil da clientela de um programa de pronto-atendimento psicológico a estudantes universitários. Psicologia em Estudo, 9(1), 47-54.

Pillon, S. C., \& Corradi-Webster, C. M. (2006). Teste de identificação de problemas relacionados ao uso de álcool entre estudantes universitários. Revista Enfermagem UERJ, 14(3), 325-332.

Pinho, R. (2016). Caracterização da clientela de um programa de atendimento psicológico a estudantes universitários. Psicología, Conocimiento y Sociedad, 6(1), 114-130.

Rocha, L. A., Lopes, A. C. F. M. M., Martelli, D. R. B., Lima, V. B., \& Martelli-Júnior, H. (2011). Consumo de álcool entre estudantes de faculdades de Medicina de Minas Gerais, Brasil. Revista Brasileira de Educação Médica, 35(3), 369-375.

Romaña M. A. (1996). Do psicodrama pedagógico à pedagogia do drama. Campinas: Papirus.

Scorsolini-Comin, F. (2014). Aconselhamento psicológico e psicoterapia: aproximações e distanciamentos. Contextos Clínicos, 7(1), 02-14.

Serpa, M. N. F., \& Santos, A. A. A. (2001). Atuação no ensino superior: um novo campo para o psicólogo escolar. Psicologia Escolar e Educacional, 5(1), 27-35.

Simão, A. M. V., Flores, A., Fernandes, S. \& Figueira, C. (2008). Tutoria no ensino superior: concepções e práticas. Revista de Ciências da Educação, 7, 75-88.

Tempsky, P. Z., \& Martins, M. A. (2012). A responsabilidade da Escola Médica na manutenção da saúde e da qualidade de vida do estudante. In: S. Baldassin (Org.). Atendimento psicológico aos estudantes de medicina. Técnica e ética. São Paulo: Edipro, p.51-58.

Troncon, L. E., Del-Bem, C. M., Machado V. F., Madisson, M. M., Resende, T. L., \& Valério, F. P. (2013). Relationship between academic performance and affective changes during the first year at medical school. Med Teach, 35(5), 404-410.

Verger, P., Guagliardo, V., Gilbert, F., Rouillon, F., \& Kovess-Masfety, V. (2010). Psychiatric disorders in students in six French universities: 12-month prevalence, comorbidity, impairment and helpseeking. Social Psychiatry and Psychiatric Epidemiology, 45(2), 189-99. 
Wagner, G. A., \& Andrade, A. G. (2008). Uso de álcool, tabaco e outras drogas entre estudantes universitários brasileiros. Revista de psiquiatria clínica, 35(1), 48-54.

Zivin, K., Eisenberg, D., Gollust, S. E., \& Golberstein, E. (2009). Persistence of mental health problems and needs in a college student population. Journal of Affective Disorders, 117(3), 180-185.

Recebido: $15 / 02 / 2018$

Reformulado 19/07/2018

Aceite Final: 07/10/2018

Sobre os autores

Karolina Murakami é Psicóloga do Centro de Apoio Educacional e Psicológico (FMRP/USP) e Mestranda do Programa de Pós Graduação em Clínica Médica (FMRP- USP), linha de pesquisa Educação em Saúde.

Gisele Curi de Barros é Psicóloga do Centro de Apoio Educacional e Psicológico (FMRP/USP), Mestre em Enfermagem Psiquiátrica pela EERP/USP.

Cristiane Martins Peres é Pedagoga e Psicopedagoga do Centro de Apoio Educacional e Psicológico (FMRP/USP), Mestre em Psicologia pela FFCLRP/USP, Doutora em Educação em Saúde pela FMRP/USP.

Rodrigo Humberto Flauzino é Pedagogo do Centro de Apoio Educacional e Psicológico (FMRP/USP) com especialização em Linguagem das Artes pelo Centro Universitário Maria Antônia/USP.

Maria de Fátima Aveiro Colares é Psicóloga do Centro de Apoio Educacional e Psicológico (FMRP/USP), Mestre e Doutora em Psicologia pela FFCLRP/USP, Pós-doutorado em Educação em Saúde pelo Departamento de Clínica Médica da FMRP/USP. 